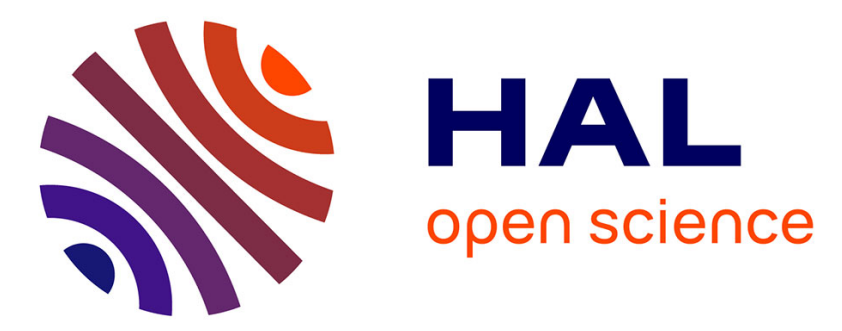

\title{
Theoretical study of optical properties of anti phase domains in GaP
}

Eric Tea, J. Vidal, Laurent Pedesseau, Charles Cornet, Jean-Marc Jancu, Jacky Even, Sana Laribi, Jean Francois Guillemoles, Olivier Durand

\section{To cite this version:}

Eric Tea, J. Vidal, Laurent Pedesseau, Charles Cornet, Jean-Marc Jancu, et al.. Theoretical study of optical properties of anti phase domains in GaP. Journal of Applied Physics, 2014, 115 (6), pp.063502. 10.1063/1.4864421 . hal-00979596

\section{HAL Id: hal-00979596 https://hal.science/hal-00979596}

Submitted on 16 Apr 2014

HAL is a multi-disciplinary open access archive for the deposit and dissemination of scientific research documents, whether they are published or not. The documents may come from teaching and research institutions in France or abroad, or from public or private research centers.
L'archive ouverte pluridisciplinaire HAL, est destinée au dépôt et à la diffusion de documents scientifiques de niveau recherche, publiés ou non, émanant des établissements d'enseignement et de recherche français ou étrangers, des laboratoires publics ou privés. 


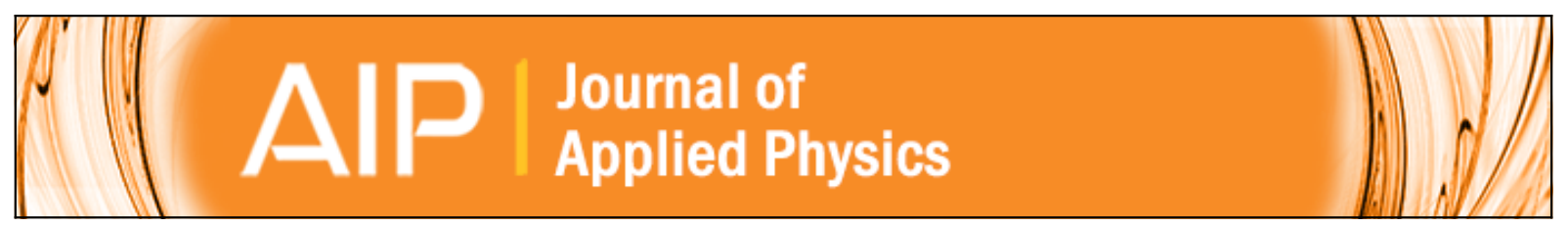

\section{Theoretical study of optical properties of anti phase domains in GaP}

E. Tea, J. Vidal, L. Pedesseau, C. Cornet, J.-M. Jancu, J. Even, S. Laribi, J.-F. Guillemoles, and O. Durand

Citation: Journal of Applied Physics 115, 063502 (2014); doi: 10.1063/1.4864421

View online: http://dx.doi.org/10.1063/1.4864421

View Table of Contents: http://scitation.aip.org/content/aip/journal/jap/115/6?ver=pdfcov

Published by the AIP Publishing

\section{Articles you may be interested in}

Verification of band offsets and electron effective masses in GaAsN/GaAs quantum wells: Spectroscopic experiment versus 10 -band $\mathrm{k} \cdot \mathrm{p}$ modeling

J. Appl. Phys. 113, 233508 (2013); 10.1063/1.4810920

First-principles study of temperature-dependent optical properties of semiconductors from ultraviolet to infrared regions

J. Appl. Phys. 111, 083508 (2012); 10.1063/1.4703922

Suppressed indium diffusion and enhanced absorption in InGaAs/GaAsP stepped quantum well solar cell Appl. Phys. Lett. 100, 053902 (2012); 10.1063/1.3681785

Studies on high resolution x-ray diffraction, optical and transport properties of In As x Sb 1 x Ga As ( $x 0.06$ ) heterostructure grown using liquid phase epitaxy

J. Appl. Phys. 96, 4989 (2004); 10.1063/1.1784620

Optical studies of GalnP(ordered)/GaAs and GalnP(ordered)/GaP/GaAs heterostructures J. Appl. Phys. 84, 2846 (1998); 10.1063/1.368426

\section{AlP Re-register for Table of Content Alerts}




\title{
Theoretical study of optical properties of anti phase domains in GaP
}

\author{
E. Tea, ${ }^{1,2, a)}$ J. Vidal, ${ }^{1}$ L. Pedesseau, ${ }^{2}$ C. Cornet, ${ }^{2}$ J.-M. Jancu, ${ }^{2}$ J. Even, ${ }^{2}$ S. Laribi, ${ }^{1}$ \\ J.-F. Guillemoles, ${ }^{1}$ and O. Durand ${ }^{2}$ \\ ${ }^{1}$ Institute of R\&D on Photovoltaic Energy (IRDEP) (UMR 7174, EDF-CNRS-ENSCP), 6 Quai Watier BP 49, \\ 78401 Chatou cedex, France \\ ${ }^{2}$ FOTON INSA-Rennes (UMR 6082 CNRS), Université Européenne de Bretagne, 20 Avenue des Buttes \\ de Cö̈smes, 35708 Rennes, France
}

(Received 6 January 2014; accepted 25 January 2014; published online 10 February 2014)

\begin{abstract}
III-V/Si heterostructures are currently investigated for silicon photonics and solar energy conversion. In particular, dilute nitride alloy GaAsPN grown on a GaP/Si platform exhibits lattice match with $\mathrm{Si}$ and an optimal band gap configuration for tandem solar cell devices. However, monolithic "coherent" growth of the GaP thin layer on Si suffers from the nucleation of extended structural defects, which can hamper device operation as well as the GaP/Si interface level and through their propagation inside the overall heterostructure. However, the effect of such structural defects on optical and transport properties is actually not well understood in details. In this letter, we investigate the anti phase domains defect (also called inversion domains) by means of $a b$ initio calculations giving insights into the alteration of optical and transport properties of GaP due to the defective GaP/Si interface. (C) 2014 AIP Publishing LLC. [http://dx.doi.org/10.1063/1.4864421]
\end{abstract}

\section{INTRODUCTION}

Lattice matched III-V/Si heterostructures have attracted a lot of attention recently since they constitute fundamental building blocks for the integration of efficient optical functions on silicon substrates. Development of energy efficient optical interconnects and ultra-fast optoelectronic devices via monolithic optoelectronic integrated circuits (OEICs) has so far driven their studies. ${ }^{1}$ III-V/Si optoelectronic devices aim at taking advantage of the excellent optical properties of III-V semiconductors, for example, fast optical data communication, thus reducing the total length of intra and inter-chip electrical interconnection. This growing research domain is known as silicon photonics and is of interest for other more recent applications, such as the target application of this article: photovoltaic solar energy conversion.

On one hand, silicon has been the material of choice for solar energy conversion because of its abundance and well controlled technology, resulting during many years in the best performance-to-cost ratio. Conventional silicon solar cells (single Si crystal) yield $\sim 25 \%$ record solar to electrical power conversion efficiencies, ${ }^{2}$ close to its $31 \%$ theoretical limit (the so-called Shockley-Queisser limit for a single junction solar cell under unconcentrated sunlight illumination ${ }^{3}$ ) despite its poor light harvesting capability originating from its indirect band gap. On the other hand, III-V compounds display tunable direct band gaps and lattice parameters, and excellent optical properties. Ultimately, single crystal GaAs solar cells hold the record conversion efficiency for single junction devices at $28.8 \%{ }^{2}$

To surpass the Shockley-Queisser limit defined for a single absorber material, novel device designs have been proposed, such as, in particular, series-connected two junction

\footnotetext{
a) Author to whom correspondence should be addressed. Electronic mail: etea.contact@gmail.com
}

solar cells, also called tandem cells. In such a structure, two absorber materials with different band gaps are stacked, and convert light from their assigned part of the solar spectrum thus reducing thermalization loss (for a description of loss mechanisms in solar cells see, e.g., Ref. 4). It has been theoretically demonstrated that a maximum $37 \%$ conversion efficiency is achievable with a GaAsPN/Si tandem cell, exceeding that of a single junction device ${ }^{5}$ while taking advantage of the well established Si technology and excellent optical properties of III-V compounds. Indeed, not only the $\mathrm{GaAs}_{0.09} \mathrm{P}_{0.87} \mathrm{~N}_{0.04}$ dilute nitride alloy is lattice matched to $\mathrm{Si}^{5,6}$ but also this GaAsPN/Si structure exhibits the optimal 1.7/1.1 eV band gap combination for a tandem cell. ${ }^{5}$ However, the structural properties of the III-V layer grown onto Si are currently the limiting factor for an optimal tandem solar cell operation.

The fabrication of an efficient monolithically integrated $\mathrm{GaAsPN} / \mathrm{Si}$ heterostructures requires defect free heteroepitaxy. Indeed, structural defects may form recombination centers leading to increased radiative loss in the solar cell, and can also degrade transport properties. Despite fulfilling lattice matching requirement, the heteroepitaxial growth of III-V polar semiconductors on a non-polar Si (001) surface can still generate detrimental structural defects. In particular, Anti Phase Domains (APDs, also called Inversion Domains) has been observed to develop in the polar region. ${ }^{6-14}$ These kind of extended structural defects nucleate at Si surface monoatomic height steps (the surface being not perfectly planar). When depositing atomic monolayers onto such a surface in a [A-B-A-B...] type sequence, the monoatomic height step induces a stacking fault, forming the Anti Phase Boundary (APB). Therefore, a [A-B-A-B...] stacking pattern on one side of the APB faces a [B-A-B-A...] pattern on the other side, and the APB is composed by A-A and B-B bonds called wrong bonds.

Various methods have been proposed to limit APD generation during the III-V growth, such as Si surface preparation 
(bi-atomic step formation by step bunching, canceling the stacking fault formation) $)^{6-8}$ and low temperature growth (favoring APB self annihilation, e.g., $\{111\}$ and $\{-111\}$ APBs running towards one another) on miscut Si (001) surfaces. ${ }^{9,10}$ A number of experimental investigations have shed some light on APB growth dynamics. ${ }^{11,12}$ However, while successful III-V on Si overgrowth has been reported, ${ }^{13-16}$ most theoretical work has been restricted to the energetical stability of such extended defects. Rubel et al. calculated APB formation energies for different plane orientations in $\mathrm{GaP}$ and GaAs, within the Density Functional Theory (DFT) framework. ${ }^{17}$ Their work based on supercell calculations suggested that $\{110\}$ APBs exhibit the lowest formation energy because $\{110\}$ APBs are stoichiometric and contain the same number of A-A and B-B wrong bonds. Hence, no excess charge is present on the APB and no extra electric field develops in the supercell, in opposition to the $\{111\}$ and $\{001\}$ cases. This result compared favorably with earlier studies based on wrong bond energy, charge transfer between wrong bonds, and wrong bond counting in GaAs. ${ }^{18}$ Indeed, A-A and B-B wrong bonds exhibit opposite excess charge and can be assimilated to acceptor and donor centers. On a $\{110\}$ APB, compensation occurs on the same APB, whereas in the non stoichiometric cases, compensation involves inter APB charge transfer or is incomplete for distant APBs. ${ }^{18,19}$ However, these studies focused on APBs formation only and optical properties were not mentioned.

Therefore, in this article, we investigate $\{110\}$ APBs in $\mathrm{GaP}$ by means of advanced $a b$ initio calculations based on many body perturbation theory. Focus is set on determining to which extent APBs will alter bulk optical and electronic properties, which are of prime importance for an optimal III-V/Si tandem solar cell operation.

\section{CALCULATION PROCEDURE}

$A b$ initio calculations on $\{110\}$ APDs of GaP were performed with the Projector Augmented Wave method (PAW) implemented in the VASP package ${ }^{20,21}$ and using the Generalized Gradient Approximation-Perdew-BurkeErnzerhof (GGA-PBE) exchange-correlation functional. Gallium $3 \mathrm{~d}$ electrons were only included in the atomic positions relaxation steps of the primitive cell and supercells. Electronic structure calculations were carried out with the perturbative one shot GW method (known as $\mathrm{G}_{0} \mathrm{~W}_{0}$ ) implemented in the VASP package. ${ }^{22-24}$ DFT has been used for the relaxation procedures, and for the calculation of wavefunctions and energies entering the construction of $\mathrm{GW}$ self-energies. The valence band splitting caused by spin-orbit coupling $\left(80 \mathrm{meV}^{25}\right)$ is relatively small compared with $\mathrm{GaP}$ band gap $\left(2.2 \mathrm{eV}^{25}\right)$ and has therefore not been accounted for in our calculations. The study of $\{110\}$ APD requiring a unit cell exhibiting a cell vector normal to $\{110\}$, the chosen unit cell vectors are given by $(1 / 2,-1 / 2,0)$, $(1 / 2,1 / 2,0)$ and $(0,0,1)$, and contains four atoms, twice the irreducible atomic pattern.

The calculated bulk GaP lattice parameter is $5.5 \AA$, $1 \%$ larger than the $0 \mathrm{~K}$ reference, ${ }^{25}$ which is typical of GGA functionals. ${ }^{26}$ The band gaps $E_{g}^{i}$ and energy separations of
TABLE I. Bulk GaP calculation results compared with experimental data compiled in Ref. 25. All energies are given in eV.

\begin{tabular}{lccc}
\hline \hline & This work $\left(\mathrm{G}_{0} \mathrm{~W}_{0}\right)$ & This work (HSE06) & Experimental data ${ }^{25}$ \\
\hline$E_{g}^{\Gamma}$ & 2.76 & 2.58 & 2.86 \\
$E_{g}^{L}$ & 2.57 & 2.43 & 2.72 \\
$E_{g}^{X}$ & 2.20 & 2.35 & 2.35 \\
$E_{C}^{L}-E_{C}^{\Gamma}$ & -0.19 & -0.15 & -0.14 \\
$E_{C}^{X}-E_{C}^{\Gamma}$ & -0.56 & -0.23 & -0.51 \\
\hline \hline
\end{tabular}

the conduction band valleys $E_{C}^{i}-E_{C}^{j}(i, j=\Gamma, L, X)$ calculated with $\mathrm{G}_{0} \mathrm{~W}_{0}$ show an overall satisfactory agreement with reference data ${ }^{25}$ and with previous GW studies. ${ }^{27}$ The use of advanced functionals, such as HSE06, did not improve the valley separations (in particular, $E_{C}^{X}-E_{C}^{\Gamma}$ ) (see Table I).

\section{RESULTS}

APDs were modeled using supercells built from $\{110\}$ four atoms unit cells stacked along the $(1 / 2,1 / 2,0)$ direction. APB spacing has been set to half the width of the supercell, so that each domain on both side of an APB has the same size (see Fig. 1(a)). Distortion of atomic positions around APBs due to wrong bonding (Ga-Ga and P-P bonds) is reproduced by atomic relaxation. The calculated shear domain displacement (one domain shifted along (001) with respect to the other) and boundary expansion (longer bond length around APBs) are, $9 \%$ and $2 \%$ of the calculated ideal Ga-P bond length, respectively, in excellent agreement with TEM
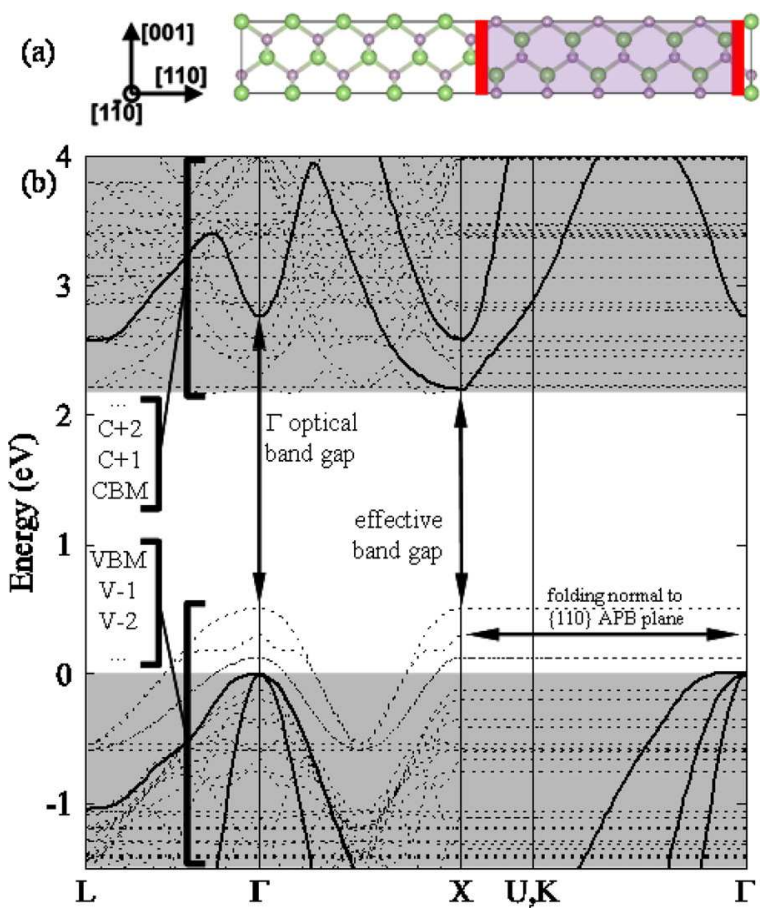

FIG. 1. (a) Sketch of a $\{110\}$ supercell containing an APD. APBs are indicated by thick vertical lines (b) Calculated bulk GaP GW band structures using the irreducible unit cell (thick solid lines), and for a $15.5 \AA \mathrm{APB}$ spacing supercell (dots). Quasi-particle energy level labeling scheme at $\Gamma$ is indicated. Shaded areas correspond to energy space occupied by bulk states. 


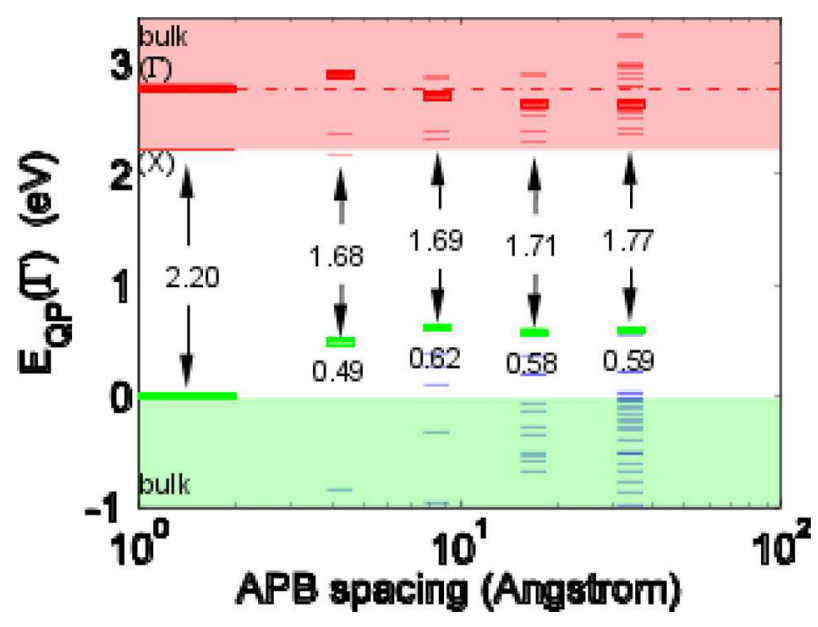

FIG. 2. Calculated quasi-particle energies at $\Gamma$ versus APB spacing are indicated by thin horizontal lines for the relaxed configurations. Thick horizontal lines indicate the calculated VBM and $\Gamma$-CBM. Shaded energy regions are populated by bulk conduction (CBM around $\mathrm{X}$ ) and valence (VBM at $\Gamma$ ) bands. Bulk GaP results are shown at zero APB spacing for comparison. Calculated effective band gaps and VBM shifts are indicated.

measurements. ${ }^{28}$ All following results refer to relaxed supercells.

Fig. 1(b) shows the calculated GW band structure for a supercell with $15.5 \AA \mathrm{APB}$ spacing along a path in reciprocal space ( $\Gamma-\mathrm{X}$ parallel to the APB, $\Gamma-\mathrm{K}$ normal to the APB). Since $\{110\}$ APBs are stoichiometric, no electric field is present. The macroscopic potentials (DFT energy reference) have therefore been averaged over the primitive and super cells and aligned, ${ }^{29}$ for bulk and APD band structures comparison. Aside the expected band folding along (001) $\Gamma-X$ direction, the band structure exhibits several striking features. (i) Band folding also occurs along the (110) $\Gamma-\mathrm{K}$ direction, due to the construction of the supercell. Indeed, the presence of APBs breaks the unit cell (110) translation symmetry and only the supercell (110) translation symmetry is conserved. The supercell being built from stacking four atoms unit cells in the (110) direction, the longer the supercell the more folding occurs. (ii) While the Conduction Band Minimum (CBM) energy seems unchanged (conduction bands within the shaded area), the Valence Band Maximum (VBM) experiences a dramatic upward shift (typically
$0.5-0.6 \mathrm{eV}$ ) leading to band gap reduction. (iii) The threefold degeneracy of bulk VBM (without spin orbit coupling) has been lifted due to symmetry breaking caused by the APD.

Calculated quasi-particle energies at $\Gamma$ for various APB spacings are plotted in Fig. 2. Their labeling scheme is indicated in Fig. 1(b) (in increasing energy order:... V-2, V-1, VBM, CBM, C $+1, \mathrm{C}+2 \ldots)$. Calculated VBM shifts with respect to the bulk VBM are indicated on Fig. 2. Focus will be put on the calculated quasi-particle energy levels at $\Gamma$ and, in particular, those located inside the bulk $\Gamma$ optical band gap shown in Fig. 1, which will be called In-Gap (IG) states.

The valence bands pushed above the bulk VBM exhibit dispersion in directions parallel to APB planes (as along the (001) $\Gamma$-X direction) and can be assimilated to surface or interface bands. However, they do not exhibit dispersion in the direction normal to the $\{110\}$ APB plane ((110) $\Gamma-\mathrm{K}$ direction) which indicates real space localization. Fig. 3(a) shows the VBM partial charge density integrated in the planes parallel to APBs for various APB spacings. APB coupling caused by finite size of supercells induces a large delocalization of wavefunctions. However, for large APB spacing, VBM partial charge density clearly reveals localization on the APB planes as the density drops between APBs. Meanwhile VBM shifts shown on Fig. 2 are found to hardly vary. Therefore, the calculated VBM shifts are attributed to the presence of the APBs themselves and not to supercell finite size effects. Fig. 3(b) shows the partial charge density for some IG states for $31.1 \AA$ APB spacing. We emphasize that these states are not related to point defects but are part of electronic bands. Valence IG states are localized on the APB planes, whereas conduction IG states exhibit a weak localization. Moreover, the calculated CBM energies are close to the bulk values, suggesting that the conduction bands may only be weakly affected by the APBs. Therefore, the CBM is most likely to originate from band folding caused by the system geometry ( $\{110\}$ unit cell building blocks). This is confirmed by inspecting the projection of wavefunctions as used in supercell unfolding procedures. ${ }^{30}$ The projection of the supercell CBM on the bulk wavefunction evaluated at $\mathrm{X}$ is larger than the same projection on the
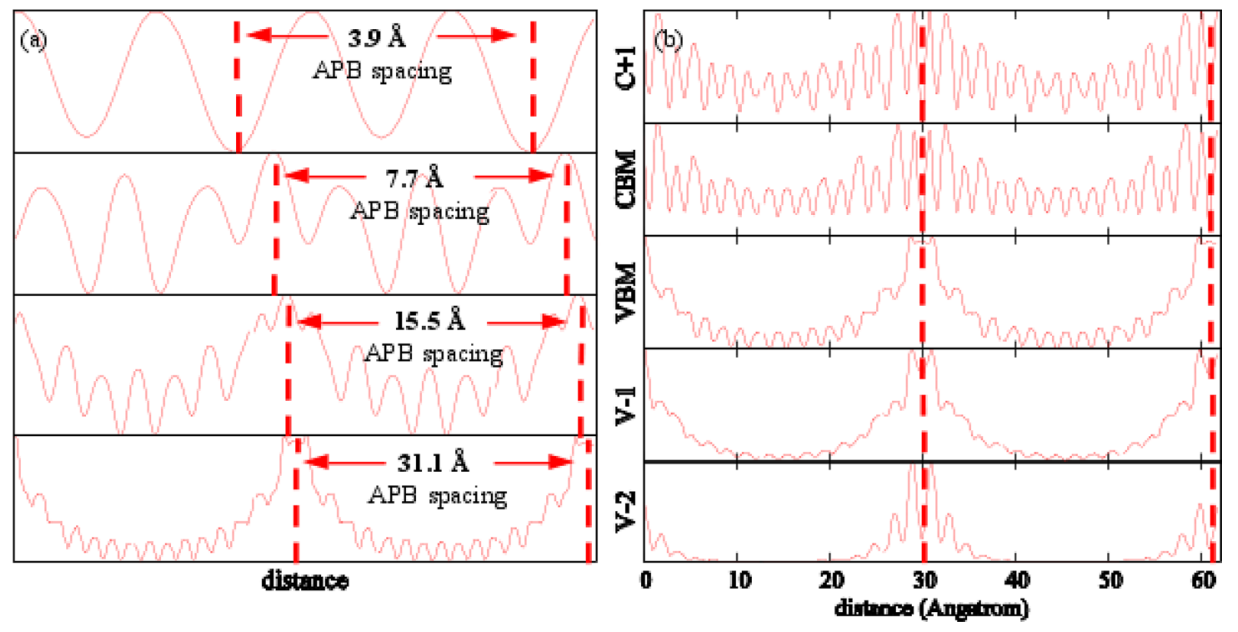

FIG. 3. IG states partial charge density evaluated at the $\Gamma$ point, integrated in the planes parallel to APBs. (a) VBM for various APB spacings. (b) Some IG states for $31.1 \AA$ APB spacing. APB positions are indicated by vertical dashed lines. 
bulk $\Gamma$ wavefunction by two orders of magnitude. However, the CBM has an X "character" because it "originates" from $\mathrm{X}$ due to band folding. Indeed, one cannot "strictly unfold" the supercell because of the breaking of the crystal symmetries caused by the APB itself. Nevertheless, for large APB spacing, one expects the material to exhibit bulk properties. Hence, the defective material should exhibit an effective indirect band gap.

To assess the nature of the calculated effective band gap, the optical properties of the IG states were investigated by the calculation of their optical coupling strength as a function of APB spacing. Results are summarized in Table II. The calculated optical coupling strengths reveal that the CBM and VBM are very weakly coupled. The strongest coupling with the VBM actually involves a higher conduction state whose energy lies the closest to the bulk one at $\Gamma$ (here called $\Gamma$-CBM, indicated by thick horizontal lines in Fig. 2, and listed in Table II). As discussed in previous paragraph, the IG conduction states have "folded" character. Therefore, they are not allowed to optically couple to the VBM which occurs at $\Gamma$. Hence, the IG conduction states should not favor radiative recombination. Wavefunction projections reveal that the $\Gamma$-CBM is actually $\Gamma$-like and do not originate from band folding. In the case of large APB spacing, one would expect to have two bulk-like domains in the supercell on each side of the APB. While APBs break the bulk crystal symmetry, the fact that the CBM do not strongly optically couple to the VBM suggests that the overall defective material (with APDs) still exhibits an indirect band gap like bulk GaP. Examination of Fig. 2 also reveals that $\Gamma$ CBM energies are actually pushed below their bulk values. Added to the VBM shifts, this further reduces the $\Gamma$ optical band gap. This band gap reduction is illustrated on Fig. 4 which shows the imaginary part of the dielectric function $\varepsilon_{i}$. These have been calculated directly from the wavefunctions by integrating the optical coupling strength over all direct optical transitions. ${ }^{31}$ The absorption onset appears at much lower photon energies in the defective material $(\sim 1.7 \mathrm{eV})$ compared with the bulk one $(\sim 2.8 \mathrm{eV})$. Photon absorption (and hence emission) becomes much stronger when photon energies are large enough to reach the $\Gamma$-CBM gaps $(\sim 2.0$ and $\sim 3.0 \mathrm{eV}$ for the defective and bulk materials, respectively), especially when reaching the bulk $\Gamma$-CBM gap energy. Examination of the amplitudes of $\varepsilon_{i}$ reveals sub band gap (bulk value) radiative loss in the defective material, but not with an enhanced character.

TABLE II. Optical coupling strength $\left\langle\varphi_{V}|-i \hbar \nabla| \varphi_{C}\right\rangle^{2}$ between valence IG states, the CBM, and the $\Gamma$-CBM for various APB spacings, in units of the optical coupling strength of bulk GaP evaluated at $\Gamma$.

\begin{tabular}{|c|c|c|c|c|c|c|}
\hline \multirow[b]{2}{*}{ APB spacing } & \multicolumn{2}{|c|}{$7.7 \AA$} & \multicolumn{2}{|c|}{$15.5 \AA$} & \multicolumn{2}{|c|}{$31.1 \AA$} \\
\hline & $\mathrm{CBM}$ & $\mathrm{C}+2$ & $\mathrm{CBM}$ & $\mathrm{C}+4$ & $\mathrm{CBM}$ & $\mathrm{C}+6$ \\
\hline$\hbar \omega$ from VBM $(\mathrm{eV})$ & 1.69 & 2.09 & 1.71 & 2.06 & 1.77 & 2.05 \\
\hline VBM & 0.01 & 0.97 & 0.00 & 0.62 & 0.04 & 0.37 \\
\hline $\mathrm{V}-1$ & 0.01 & 0.86 & 0.01 & 0.72 & 0.01 & 0.35 \\
\hline $\mathrm{V}-2$ & 0.01 & 0.69 & 0.00 & 0.58 & 0.02 & 0.31 \\
\hline
\end{tabular}

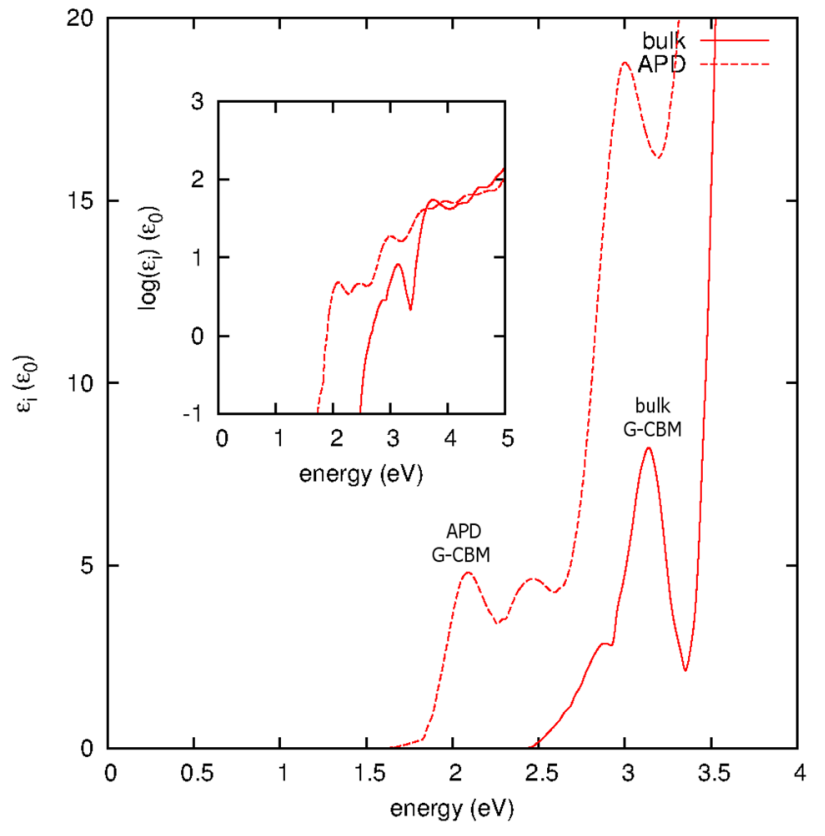

FIG. 4. Imaginary part of the calculated dielectric function for direct optical transitions $\varepsilon_{i}(q \rightarrow 0, \omega)$. Results for bulk and defective materials are drawn with solid and dashed lines, respectively. The inset shows the same data on a $\log$ scale.

Localization of the IG valence partial charge density (over a few atomic layers, as shown on Fig. 3(b)) suggests that charge carriers can be trapped on the APB planes, which may be detrimental to solar cell operation. However, APBs are not point defects, but structural planar defects. Therefore, transport still occurs in APB planes. In the presence of APDs, an effective mass for the VBM $(\Gamma-\mathrm{X}$ direction) could then be calculated $m_{001}^{A P D}=0.72 m_{0}$ and found to be larger than the bulk value $m_{001}^{\text {bulk }}=0.48 m_{0}$. This suggests that APBs can be assimilated to quantum wells, forming planar conduction channels for holes. Moreover, for small APB spacing, APB coupling induces inter APB charge transfer. Therefore, transport across APBs can take place for very high APD densities. In any case, the VBM experiences a large upward shift $(0.5-0.6 \mathrm{eV})$ compared with bulk $\mathrm{GaP}$, and should be considered for band alignment purposes and hole collection.

\section{CONCLUSION}

In this article, we reported GW calculations of optoelectronic properties of $\{110\}$ APDs in GaP. The nature of the effective band gap shows a strong indirect character. Reduction of the indirect band gap has been evidenced, due to a large $(0.5-0.6 \mathrm{eV}) \mathrm{VBM}$ upward shift. Reduction of the $\Gamma$ optical band gap caused by the VBM upward shift and a small $\Gamma$-CBM downward shift has also been evidenced. Optical coupling strength calculation revealed that the VBM and CBM are only weakly coupled. Calculation of the imaginary part of the dielectric function for direct optical transitions did not show enhanced sub band gap radiative coupling. The VBM partial charge density is localized on the APB planes, therefore they can trap charge carriers. However, transport in the APB planes can take place, and is 
characterized by an effective mass larger than that of bulk. APBs can be assimilated to effective quantum wells forming planar conduction channels for holes. On the other hand, the CBM wavefunction is mainly delocalized and bulk-like.

\section{ACKNOWLEDGMENTS}

The authors acknowledge the ANR projects SINPHONIC (Grant No. 2011 JS03 006-01) and MENHIRS (Grant No. ANR-2011-PRGE-007-0) for their financial support to this research.

${ }^{1}$ B. Kunert, K. Volz, and W. Stolz, Phys. Status Solidi B 244, 2730 (2007).

${ }^{2}$ M. A. Green et al., Prog. Photovoltaics 21, 827 (2013).

${ }^{3}$ W. Shockley and H. Queisser, J. Appl. Phys. 32, 510 (1961).

${ }^{4}$ L. C. Hirst and N. J. Ekins-Daukes, Prog. Photovoltaics 19, 286 (2011).

5 J. F. Geisz and D. J. Friedman, Semicond. Sci. Technol. 17, 769 (2002).

${ }^{6}$ B. Kunert, I. Nemeth, S. Reinhard, K. Volz, and W. Stolz, Thin Solid Films 517, 140 (2008).

${ }^{7}$ T. J. Grassman, M. R. Brenner, S. Rajagopalan, R. Unocic, R. Dehoff, M. Mills, H. Fraser, and S. A. Ringel, Appl. Phys. Lett. 94, 232106 (2009).

${ }^{8}$ K. Volz, A. Beyer, W. Witte, J. Ohlmann, I. Németh, B. Kunert, and W. Stolz, J. Cryst. Growth 315, 37 (2011).

${ }^{9}$ H. Yonezu, Semicond. Sci. Technol. 17, 762 (2002).

${ }^{10}$ Y. Takagi, H. Yonezu, K. Samonji, T. Tsuji, and N. Ohshima, J. Cryst. Growth 187, 42 (1998).

${ }^{11}$ A. Létoublon, W. Guo, C. Cornet, A. Boulle, M. Véron, A. Bondi, O. Durand, T. Rohel, O. Dehaese, N. Chevalier, N. Bertru, and A. Le Corre, J. Cryst. Growth 323, 409 (2011).
${ }^{12}$ W. Guo, A. Bondi, C. Cornet, A. Létoublon, O. Durand, T. Rohel, S. Boyer-Richard, N. Bertru, S. Loualiche, J. Even, and A. Le Corre, Appl. Surf. Sci. 258, 2808 (2012)

${ }^{13}$ Y. Furukawa, H. Yonezu, A. Wakahara, S. Ishiji, S. Y. Moon, and Y. Morisaki, J. Cryst. Growth 300, 172 (2007).

${ }^{14}$ T. Nguyen Thanh, C. Robert, W. Guo, A. Létoublon, C. Cornet, G. Elias, A. Ponchet, T. Rohel, N. Bertru, A. Balocchi, O. Durand, J. S. Micha, M. Perrin, S. Loualiche, X. Marie, and A. Le Corre, J. Appl. Phys. 112, 053521 (2012).

${ }^{15}$ S. Almosni, C. Robert, T. Nguyen Thanh, C. Cornet, A. Létoublon, T. Quinci, C. Levallois, M. Perrin, J. Kuyyalil, L. Pedesseau, A. Balocchi, P. Barate, J. Even, J. M. Jancu, N. Bertru, X. Marie, O. Durand, and A. Le Corre, J. Appl. Phys. 113, 123509 (2013).

${ }^{16}$ T. Quinci, J. Kuyyalil, T. Nguyen Thanh, Y. Ping Wang, S. Almosni, A. Létoublon, T. Rohel, K. Tavernier, N. Chevalier, O. Dehaese, N. Boudet, J. F. Bérar, S. Loualiche, J. Even, N. Bertru, A. Le Corre, O. Durand, and C. Cornet, J. Cryst. Growth 380, 157 (2013).

${ }^{17}$ O. Rubel and S. D. Baranovskii, Int. J. Mol. Sci. 10, 5104 (2009).

${ }^{18}$ W. R. L. Lambrecht, C. Amador, and B. Segall, Phys. Rev. Lett. 68, 1363 (1992).

${ }^{19}$ D. Vanderbilt and C. Lee, Phys. Rev. B 45, 11192 (1992).

${ }^{20}$ G. Kresse and J. Furthmüller, Phys. Rev. B 54, 11169 (1996).

${ }^{21}$ G. Kresse and D. Joubert, Phys. Rev. B 59, 1758 (1999).

${ }^{22}$ M. Shishkin and G. Kresse, Phys. Rev. B 74, 035101 (2006).

${ }^{23}$ L. Hedin, Phys. Rev. 139, A796 (1965).

${ }^{24}$ W. G. Aulbur, L. Jönsson, and J. W. Wilkins, Solid State Phys. 54, 1 (2000).

${ }^{25}$ I. Vurgaftman, J. R. Meyer, and L. R. Ram-Mohan, J. Appl. Phys. 89, 5815 (2001)

${ }^{26}$ V. Ozolins and M. Korling, Phys. Rev. B 48, 18304 (1993).

${ }^{27}$ X. Zhu and S. G. Louie, Phys. Rev. B 43, 14142 (1991).

${ }^{28}$ D. Cohen and C. B. Carter, J. Microsc. 208, 84 (2002).

${ }^{29}$ C. G. Van de Walle and R. M. Martin, Phys. Rev. B 35, 8154 (1987).

${ }^{30}$ V. Popescu and A. Zunger, Phys. Rev. B 85, 085201 (2012).

${ }^{31}$ M. L. Cohen and V. Heine, Solid State Phys. 24, 37 (1970). 